\title{
Glioblastoma of the cerebellopontine angle and internal auditory canal mimicking a peripheral nerve sheath tumor: case report
}

\author{
Hirokazu Takami, MD, PhD, ${ }^{1}$ Christoph M. Prummer, MD, ${ }^{2}$ Christopher S. Graffeo, MD, ${ }^{1}$ \\ Maria Peris-Celda, MD, PhD, ${ }^{1}$ Caterina Giannini, MD, PhD, ${ }^{3}$ Colin L. Driscoll, MD, ${ }^{2}$ and \\ Michael J. Link, MD ${ }^{1,2}$
}

Departments of ${ }^{1}$ Neurologic Surgery, ${ }^{2}$ Otorhinolaryngology-Head and Neck Surgery, and ${ }^{3}$ Laboratory Medicine and Pathology, Mayo Clinic, Rochester, Minnesota

\begin{abstract}
Glioblastoma (GBM) of the internal auditory canal (IAC) is exceedingly rare, with only 3 prior cases reported in the literature. The authors present the fourth case of cerebellopontine angle (CPA) and IAC GBM, and the first in which the lesion mimicked a vestibular schwannoma (VS) early in its natural history. A 55 -year-old man presented with tinnitus, hearing loss, and imbalance. MRI identified a left IAC/CPA lesion measuring $8 \mathrm{~mm}$, most consistent with a benign VS. Over the subsequent 4 months he developed facial weakness. The tumor grew remarkably to $24 \mathrm{~mm}$ and surgery was recommended; the main preoperative diagnosis was malignant peripheral nerve sheath tumor (MPNST). Resection proceeded via a translabyrinthine approach with resection of cranial nerves VII and VIII, followed by facial-hypoglossal nerve anastomosis. Intraoperative frozen section suggested malignant spindle cell neoplasm, but final histopathological and molecular testing confirmed the lesion to be a GBM. The authors report the first case in which absence of any brainstem interface effectively excluded a primary parenchymal tumor, in particular GBM, from the differential diagnosis. Given the dramatic differences in treatment and prognoses between malignant glioma and MPNST, this case emphasizes the importance of surgical intervention on an aggressively growing lesion, which provides both the best probability of local control and the critical tissue diagnosis.
\end{abstract}

https://thejns.org/doi/abs/10.3171/2018.8.JNS181702

KEYWORDS internal auditory canal; cerebellopontine angle; glioma; glioblastoma; oncology

$\mathrm{G}$ LIOBLASTOMA (GBM) is the most common primary CNS tumor in adults, comprising $15 \%-20 \%$ of intracranial tumors. ${ }^{6,15}$ The overwhelming majority of these tumors arise supratentorially, within the cerebral hemispheres. ${ }^{15} \mathrm{GBM}$ is uncommon within the posterior fossa and cerebellopontine angle (CPA). CPA GBMs are exceedingly rare and typically represent exophytic lesions from the cerebellar peduncle, whereas an internal auditory canal (IAC) GBM is extraordinarily rare, with only 3 prior cases reported in the literature..$^{3,17,18} \mathrm{We}$ report the fourth instance of IAC/CPA GBM, characterized by a novel presentation in which the tumor very closely mimicked a tumor of cranial nerve $(\mathrm{CN})$ sheath origin, initially diagnosed elsewhere as a benign vestibular schwannoma (VS), then subsequently a malignant peripheral nerve sheath tumor (MPNST), before ultimately being proven to be a GBM of the CPA and IAC.

\section{Case Report}

History and Examination

A 55-year-old man presented to his local physician with vertigo and persistent imbalance. Gadolinium-enhanced MRI of the brain showed an enhancing mass within the right IAC demonstrating minimal CPA extension to a maximal diameter of $8 \mathrm{~mm}$ parallel to the petrous temporal bone, most consistent with VS (Fig. 1A). There was no contact with the brainstem. According to the patient,

ABBREVIATIONS $\mathrm{CN}=$ cranial nerve; $\mathrm{CnLOH}=$ copy neutral loss of heterozygosity; $\mathrm{CPA}$ = cerebellopontine angle; $\mathrm{GBM}$ = glioblastoma; GFAP = glial fibrillary acidic protein; HB = House-Brackmann; IAC = internal auditory canal; MPNST = malignant peripheral nerve sheath tumor; SRS = stereotactic radiosurgery; VS = vestibular schwannoma.

SUBMITTED June 18, 2018. ACCEPTED August 3, 2018.

INCLUDE WHEN CITING Published online December 21, 2018; DOI: 10.3171/2018.8.JNS181702. 
management options including observation, stereotactic radiosurgery (SRS), and microsurgical resection were discussed and he elected to observe the tumor.

Within 3 months, the patient developed marked imbalance with frequent falls, as well as a new, rapidly advancing right-sided facial nerve weakness that appeared 2 months after initial evaluation and had progressed to complete paralysis (e.g., House-Brackmann [HB] grade VI) by the 4-month clinical follow-up. MRI at that time demonstrated new macrocystic/necrotic changes, and marked enlargement to $24 \mathrm{~mm}$ parallel to the petrous temporal bone with significant expansion of the CPA component, resulting in frank brainstem compression and edema (Fig. $1 \mathrm{~B}$ and $\mathrm{C}$ ). The patient self-referred to our tertiary care institution for further workup and treatment recommendations.

At the time of our initial assessment, the physical examination was significant for right-sided HB grade VI weakness, right lateral gaze nystagmus, right trigeminal sensory loss, right-sided deafness, and no lower CN dysfunction. The Romberg test was positive, and gait was wide-based and ataxic, with assistance required to prevent falling during tandem walk trials.

Due to the aggressive imaging and clinical characteristics, MPNST was considered the most likely diagnosis. Urgent surgery was recommended, including an aggressive attempt at gross-total resection, with planned resection of the facial and vestibulocochlear nerves if clearly involved and a malignancy was discovered on intraoperative frozen section pathology, followed by facial-hypoglossal nerve reanimation. The patient agreed, and was taken to surgery the next day.

\section{Intraoperative Course}

A translabyrinthine approach to the CPA was selected, which proceeded in the usual fashion. During mastoid drilling, care was taken to identify and decompress the full descending portion of the facial nerve. Within the middle ear, frank tumor was observed extending along the tympanic segment of the facial nerve and abutted the stapes. Multiple intraoperative biopsy samples were sent for frozen section to provide a distal margin, and the findings were described as a malignant spindle cell tumor of undetermined origin. The tumor had expanded the labyrinthine segment of the facial nerve as well as the geniculate ganglion. The facial nerve was transected at the second genu, and tumor resection proceeded along the proximal course of the nerves through the IAC, where it was noted to be adherent to the dura and surrounded by thickened arachnoid at the porus. Within the CPA, the tumor was debulked piecemeal. At the brainstem interface, multiple areas of infiltration into the brainstem were observed and biopsy confirmed tumor; however, resection could not safely proceed into the brainstem at those locations, and a near-total resection was completed with all other visible tumor removed (99\% resection).

\section{Postoperative Course}

Following tumor resection, attention was turned to the facial reanimation procedure, and the hypoglossal nerve was found in its extracranial descending segment, where
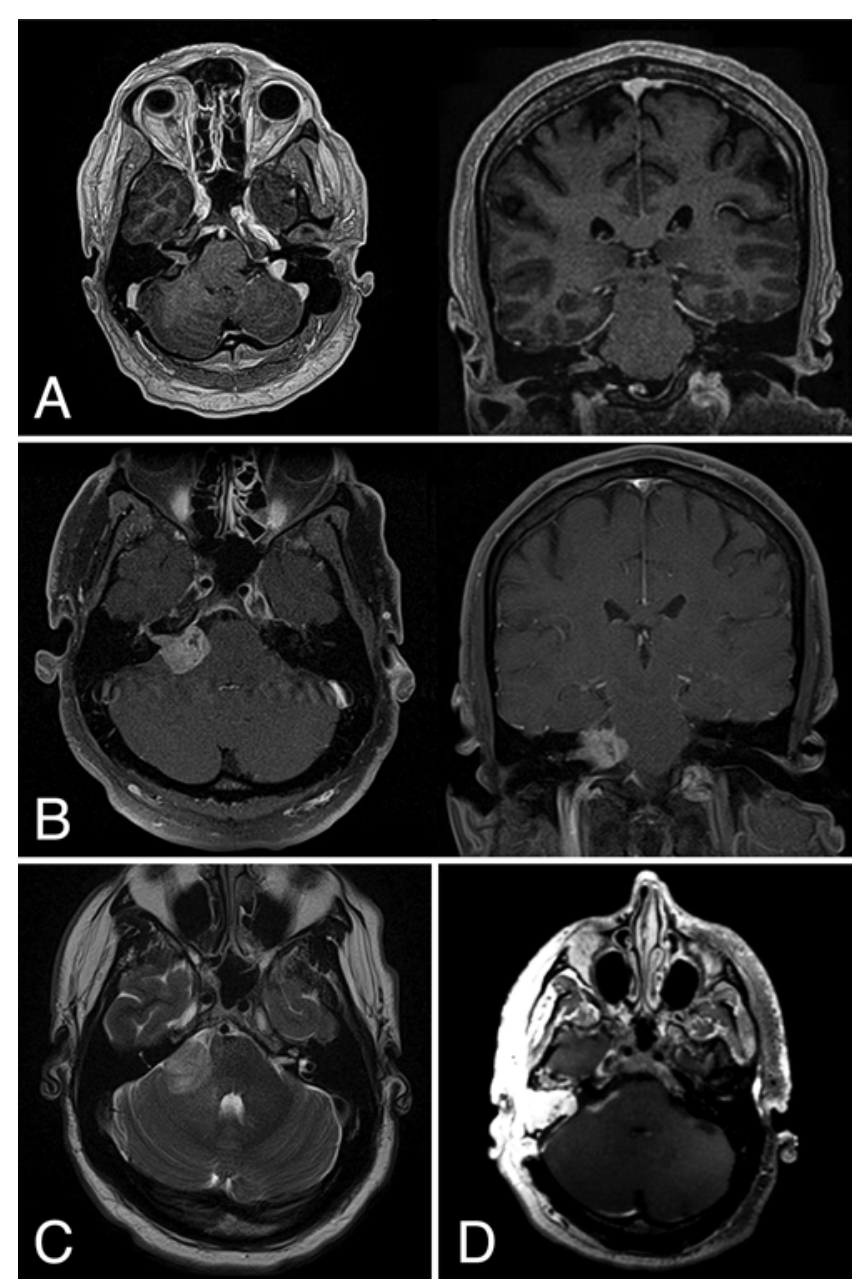

FIG. 1. A: Gadolinium-enhanced magnetization-prepared rapid gradient echo MRI sequence of the brain in the axial and coronal planes demonstrated an enhancing lesion seated at the IAC with minimal extension into the CPA and a maximum extracanalicular diameter of $8 \mathrm{~mm}$, most consistent with a small VS. B: Interval follow-up T1-weighted contrastenhanced axial and coronal images acquired at 4 months demonstrated marked tumor growth to a maximal diameter of $24 \mathrm{~mm}$. C: Axial T2weighted image reveals new mass effect and edema of the middle and inferior cerebellar peduncles. D: Gadolinium-enhanced T1-weighted axial MRI obtained 1 month postoperatively at the time of radiotherapy planning identified a thin rim of superficial enhancement overlying the right lateral pons.

the nerve was transected and rotated cranially to the stylomastoid region. ${ }^{5}$ A direct end-to-end facial-hypoglossal anastomosis was performed with interrupted 9-0 nylon suture. Postoperatively, the patient recovered well, with expected unilateral hypoglossal nerve dysfunction. Histopathological analysis demonstrated a highly cellular malignant neoplasm with abundant mitoses, focal microvascular changes, and necrosis (Fig. 2A). Immunohistochemical stains showed diffuse $\mathrm{S} 100$ and glial fibrillary acidic protein (GFAP) positivity (Fig. 2B and C). H3K27me3 expression, often lost in MPNST, was retained in the tumor (Fig. 2D). Olig2, SOX10, Synaptophysin, MelanA, collagen IV, IDH1R132H, and BRAFV600E were negative. A targeted neurooncology 50-gene next-generation sequenc- 


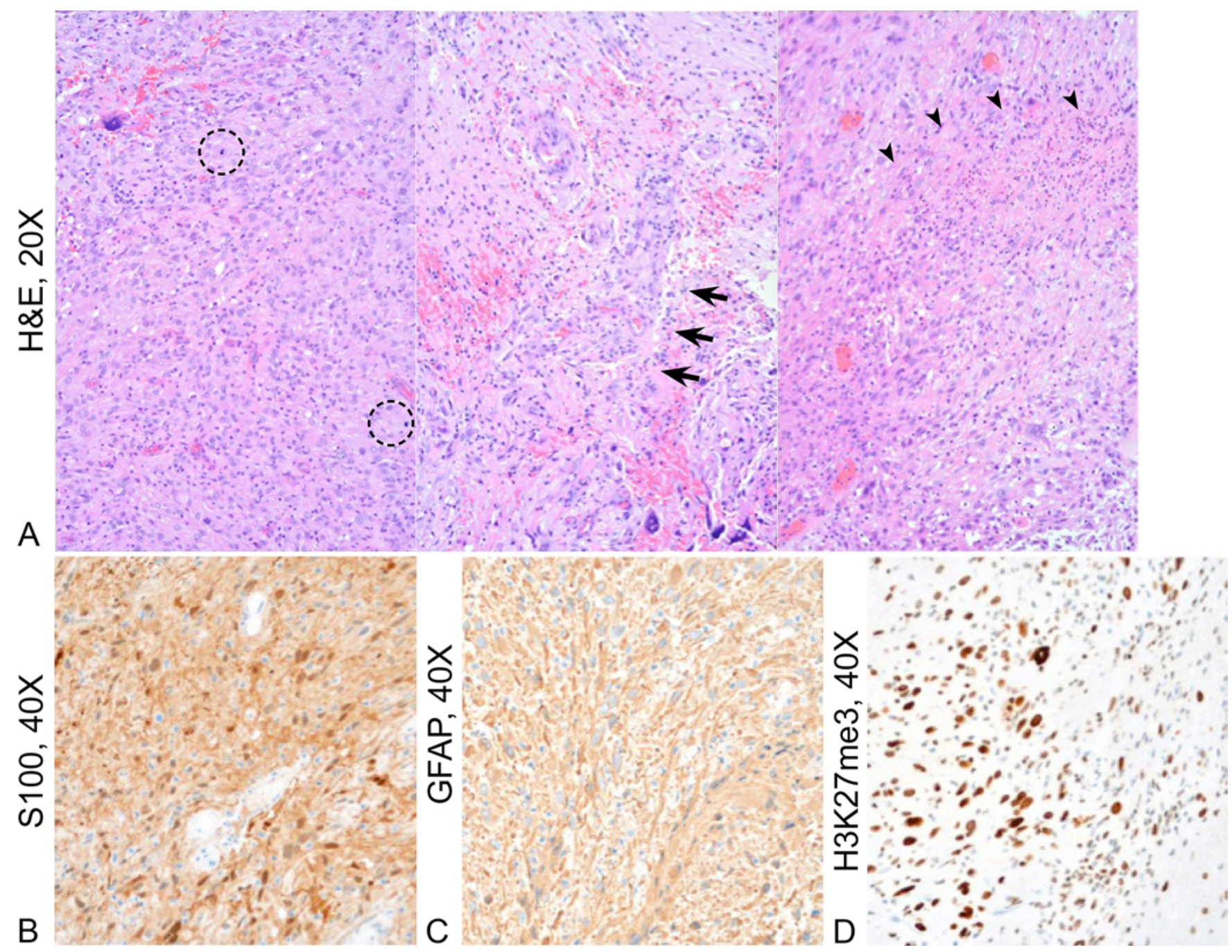

FIG. 2. H\&E-stained thin sections (A) demonstrated an undifferentiated malignant neoplasm with abundant mitoses (circles), focal microvascular changes (arrows), and necrosis (arrowheads). Immunohistochemistry identified diffuse positivity to S100 (B) and GFAP (C). Tumor cells were positive for H3K27me3 (D). Figure is available in color online only.

ing assay identified abnormalities in TERT (C250T), TP53 (c.659A > G), NFI (c.480-1G > A), RB1 (c.951_954del), and PIK3Rl (c.1126G > A). Chromosomal microarray showed a complex molecular karyotype with loss of chromosomes $2,4,8,10,11,12,14,16,18$, and 22, copy neutral loss of heterozygosity (cnLOH) of chromosome 5 , loss of 13q13.3q32.1 (including RB1), cnLOH of chromosome 17 (including TP53), cnLOH of chromosome 19, and gain of chromosome 21 , with a pattern suggestive of a hyperhaploid tumor, as previously described in a diffuse astrocytoma and a few other tumors. ${ }^{6,16}$ The histopathological features, immunohistochemical pattern of stain, and molecular features supported the diagnosis of GBM, isocitrate dehydrogenase-wildtype over MPNST. The tumor did not show MGMT promoter methylation.

Protocol-based adjuvant treatment for GBM was subsequently initiated, including temozolomide and fractionated external beam radiation therapy to a total dose of 60 Gy. MRI of the brain obtained 1 month postoperatively for radiotherapy planning purposes identified a thin rim of new enhancement appreciable on the right anterolateral surface of the pons, presumed to be recurrence or progression of retrograde tumor extension (Fig. 1D). As of the last follow-up evaluation at 5 months, the patient is neurologically stable, with no significant radiographic change in the appearance of the tumor residuum.

\section{Discussion}

GBM is the most common primary CNS tumor, arising far more frequently in the supratentorial space than in the posterior fossa, where an uncommon GBM might arise in the cerebellum (3\% of all intracranial GBMs) or brainstem. ${ }^{7}$ Furthermore, primary CNS lesions are particularly rare in the CPA, where they account for less than $2 \%$ of all neoplasms, dwarfed by VSs and other $\mathrm{CN}$ sheath tumors (80\%), meningiomas (10\%), epidermoids (6\%), and a range of rare $(<1 \%)$ neoplasms including MPNSTs, exophytic 
Takami et al.

TABLE 1. Studies of CPA GBMs extending to the IAC

\begin{tabular}{|c|c|c|c|c|c|c|c|c|c|c|c|}
\hline \multirow{2}{*}{$\begin{array}{l}\text { Authors } \\
\& \text { Year }\end{array}$} & \multirow{2}{*}{$\begin{array}{l}\text { Pt Age } \\
\text { (yrs), Sex }\end{array}$} & \multirow[b]{2}{*}{ Sxs } & \multirow{2}{*}{$\begin{array}{l}\text { Sx Duration } \\
\text { (mos) }\end{array}$} & \multicolumn{2}{|c|}{ Size (mm) } & \multirow{2}{*}{$\begin{array}{l}\text { Mos Btwn } \\
2 \text { Images }\end{array}$} & \multirow{2}{*}{$\begin{array}{l}\text { Tumor } \\
\text { Origin }\end{array}$} & \multirow[b]{2}{*}{ Chemo } & \multirow[b]{2}{*}{$\mathrm{RT}$} & \multirow{2}{*}{$\begin{array}{c}\mathrm{Ki}-67 \\
(\%)\end{array}$} & \multirow{2}{*}{$\begin{array}{c}\mathrm{FU} \\
\text { (mos) }\end{array}$} \\
\hline & & & & Initial & Last & & & & & & \\
\hline $\begin{array}{c}\text { Swaroop \& } \\
\text { Whittle, } \\
1997\end{array}$ & $22, \mathrm{M}$ & $\begin{array}{l}\text { Ear pain, gait instability, } \\
\text { nystagmus, facial } \\
\text { palsy, hearing loss, } \\
\text { decreased gag reflex }\end{array}$ & 12 & ND & NA & NA & Pons & No & Yes & ND & 12 \\
\hline $\begin{array}{l}\text { Wu et al., } \\
2011\end{array}$ & $60, M$ & $\begin{array}{l}\text { Facial numbness, } \\
\text { facial palsy, hearing } \\
\text { loss, dysarthria, } \\
\text { dysphagia }\end{array}$ & 2 & $36 \times 35 \times 33$ & NA & NA & CN VIII & No & No & $40-50$ & 2 \\
\hline $\begin{array}{l}\text { Duan et } \\
\text { al., } \\
2016^{*}\end{array}$ & $71, \mathrm{~F}$ & $\begin{array}{l}\mathrm{HA}, \mathrm{N} / \mathrm{V} \text {, altered } \\
\text { consciousness, diz- } \\
\text { ziness, nystagmus, } \\
\text { facial palsy, tinnitus }\end{array}$ & 6 & Undetectable & $36 \times 41$ & 4 & $\begin{array}{l}\text { Possibly } \\
\text { cere- } \\
\text { bellum }\end{array}$ & TMZ & $60 \mathrm{~Gy}$ & 40 & 6 \\
\hline $\begin{array}{l}\text { Present } \\
\text { study }\end{array}$ & $55, \mathrm{M}$ & $\begin{array}{l}\text { Imbalance, hearing } \\
\text { loss, tinnitus }\end{array}$ & 19 & $8 \times 14$ & $24 \times 22$ & 4 & $\begin{array}{l}\text { Possibly } \\
\text { CN VIII }\end{array}$ & TMZ & $60 \mathrm{~Gy} / 30 \mathrm{Fr}$ & ND & 3 \\
\hline
\end{tabular}

Chemo = chemotherapy; FU = follow-up; HA = headache; $N A=$ not applicable; $N D=$ no data; N/V = nausea/vomiting; pt = patient; RT = radiation therapy; Sx = symptom; TMZ = temozolomide.

All patients underwent subtotal resection, except the present case (near-total resection).

* The patient in the study of Duan et al. suffered gliosarcoma postoperatively.

metastases, lipomas, hemangiomas, paragangliomas, and choroid plexus tumors. ${ }^{4}$

Three preceding case reports have described GBM or gliosarcoma within the IAC (Table 1)., 3,17,18 These authors suggested that tumors arose from the cerebellum, pons, and CN VIII, based on their intraoperative and imaging findings. However, review of the published radiographic images demonstrates that, in each case, the tumors had wide attachment to the petrous bone, as well as clear cerebellar components, as opposed to the discrete nature of the initial lesion we identified with no discernable intraaxial attachment. Combined with the heterogeneous enhancement pattern, characteristic "ice cream cone" morphology, and extension throughout the IAC, this initial imaging finding strongly suggested VS as the underlying diagnosis. ${ }^{10}$ Similarly, upon progression, the rapid growth highlighted the possibility of a malignant process, but the character of the mass lesion's expansion as it seemed to blossom outward from the IAC was again much more consistent with a CN-based tumor such as an MPNST, but not characteristic of GBM in any way apart from its aggressiveness, or the possible presence of necrosis, both nonspecific features. Interestingly, tumor hemorrhage and/or central necrosis appear to be common features in the rare glial CPA tumors previously reported, two of which were identified as having symptomatic bleeding events, one of which prompted emergency surgical intervention. . $^{3,9}$

Although no definitive mechanistic data are available, based on the present case and the preceding literature, we speculate that there are two potential cells of origin from which these extremely rare GBMs have arisen. Heterotopic glioneuronal cell nests have been described in all components of the CNS, and are considered to be the source of primary gliomatosis; and glioneuronal heterotopias have also been reported as rare lesions in the CPA. ${ }^{12}$ Correspondingly, microscopic or macroscopic glioneu- ronal heterotopias may provide a plausible origin for the development of a GBM that is otherwise disconnected from all CNS tissue. ${ }^{2}$ Glioneuronal heterotopias are usually incidentally discovered and there is no evidence to support that there is a higher risk of malignant transformation of this abnormally located glioneuronal tissue. Alternatively, histological analyses have demonstrated that the most proximal $\mathrm{CN}$ segments adjacent to the brainstem root entry zones are covered with glial cells including astrocytes and/or oligodendrocytes, which myelinate the nerve segment just proximal to the first myelinating peripheral Schwann cells. Of particular interest, this region of oligodendrocyte-predominant myelination has been reported to be the longest on CN VIII, and one can imagine a tumor growing along the axonal trajectory from the root entry zone out to the IAC, uncannily mimicking VS in the process. ${ }^{1,8,13}$

The implications of diagnosing GBM where VS or MPNST was suspected are considerable, with ramifications in natural history, treatment, and anticipated response to therapy. Whereas VS in most circumstances can be observed, resected, or treated using SRS, GBM treatment protocols are almost universally built around a foundational triad of maximal surgical cytoreduction, conventional radiotherapy, and chemotherapy with temozolomide. ${ }^{14,15}$ In the present case, although VS was initially suspected, at the time of surgery a malignant process such as MPNST or sarcoma was considered more likely, and so a maximal tumor resection was completed, including facial and vestibulocochlear nerve sacrifice with facial reanimation surgery during the same anesthetic. Postoperatively, the final integrated diagnosis was confirmed prior to adjuvant therapy, and so the patient was initiated on standard-protocol GBM chemoradiation. Our case represents the first instance of molecular profiling in IAC or CPA GBM, demonstrating mutations in TP53, TERT, NF1, 
and $R B 1$ (among others) via targeted next-generation sequencing. Further case accumulation is required to better characterize these rare lesions, an undertaking that will benefit substantially from additional molecular studies, as the identification of any distinct mutation profiles related to epigenetic dysregulation would be of potential benefit in better establishing prognosis and treatment guidelines in a very low-incidence disease. ${ }^{11}$

GBM in the IAC or CPA is extremely rare, and has the capacity to remarkably mimic the more commonplace and benign tumors typically encountered in this location. Our case is particularly noteworthy as the first reported example of GBM located discretely in the IAC, mimicking VS on initial presentation, and then MPNST on follow-up imaging. Considering the malignant nature of GBM, early pathological diagnosis with maximal resection followed by chemotherapy and radiation therapy should be expedited to improve prognosis wherever possible. Our case demonstrates, via one extreme example, the importance of maintaining a high index of suspicion for atypical lesions, particularly in the setting of unusual imaging or clinical findings.

\section{References}

1. Bridger MW, Farkashidy J: The distribution of neuroglia and Schwann cells in the 8th nerve of man. J Laryngol Otol 94:1353-1362, 1980

2. Cooper IS, Kernohan JW: Heterotopic glial nests in the subarachnoid space; histopathologic characteristics, mode of origin and relation to meningeal gliomas. J Neuropathol Exp Neurol 10:16-29, 1951

3. Duan H, Kitazawa K, Yako T, Ichinose S, Kobayashi S, Sudo $\mathrm{M}$ : Gliosarcoma in the cerebellopontine angle with rapid tumor growth and intratumoral hemorrhage. World Neurosurg 92:580.e17-580.e21, 2016

4. Graffeo CS, Perry A, Copeland WR III, Giannini C, Neff BA, Driscoll CL, et al: Synchronous tumors of the cerebellopontine angle. World Neurosurg 98:632-643, 2017

5. Iaconetta G, Solari D, Villa A, Castaldo C, Gerardi RM, Califano G, et al: The hypoglossal nerve: anatomical study of its entire course. World Neurosurg 109:e486-e492, 2018

6. Jenkins RB, Kimmel DW, Moertel CA, Schultz CG, Scheithauer BW, Kelly PJ, et al: A cytogenetic study of 53 human gliomas. Cancer Genet Cytogenet 39:253-279, 1989

7. Jeswani S, Nuño M, Folkerts V, Mukherjee D, Black KL, Patil CG: Comparison of survival between cerebellar and supratentorial glioblastoma patients: surveillance, epidemiology, and end results (SEER) analysis. Neurosurgery 73:240-246, 2013

8. Kakita A, Wakabayashi K, Takahashi H, Ohama E, Ikuta F, Tokiguchi S: Primary leptomeningeal glioma: ultrastructural and laminin immunohistochemical studies. Acta Neuropathol 83:538-542, 1992
9. Matsuda M, Onuma K, Satomi K, Nakai K, Yamamoto T, Matsumura A: Exophytic cerebellar glioblastoma in the cerebellopontine angle: case report and review of the literature. J Neurol Surg Rep 75:e67-e72, 2014

10. Nagatani K, Takeuchi S, Otani N, Nawashiro H: Glioblastoma with dural tail. J Neurosurg 115:668, 2011

11. Nomura M, Mukasa A, Nagae G, Yamamoto S, Tatsuno K, Ueda H, et al: Distinct molecular profile of diffuse cerebellar gliomas. Acta Neuropathol 134:941-956, 2017

12. Peris-Celda M, Giannini C, Diehn FE, Eckel LJ, Neff BA, Van Gompel JJ: Glioneuronal heterotopia presenting as a cerebellopontine angle tumor of the cranial nerve VIII, case report. World Neurosurg 114:289-292, 2018

13. Reifenberger G, Boström J, Bettag M, Bock WJ, Wechsler W, Kepes JJ: Primary glioblastoma multiforme of the oculomotor nerve. Case report. J Neurosurg 84:1062-1066, 1996

14. Sanai N, Polley MY, McDermott MW, Parsa AT, Berger MS: An extent of resection threshold for newly diagnosed glioblastomas. J Neurosurg 115:3-8, 2011

15. Stupp R, Mason WP, van den Bent MJ, Weller M, Fisher $\mathrm{B}$, Taphoorn MJ, et al: Radiotherapy plus concomitant and adjuvant temozolomide for glioblastoma. $\mathbf{N}$ Engl J Med 352:987-996, 2005

16. Sukov WR, Ketterling RP, Wei S, Monaghan K, Blunden P, Mazzara P, et al: Nearly identical near-haploid karyotype in a peritoneal mesothelioma and a retroperitoneal malignant peripheral nerve sheath tumor. Cancer Genet Cytogenet 202:123-128, 2010

17. Swaroop GR, Whittle IR: Exophytic pontine glioblastoma mimicking acoustic neuroma. J Neurosurg Sci 41:409-411, 1997

18. Wu B, Liu W, Zhu H, Feng H, Liu J: Primary glioblastoma of the cerebellopontine angle in adults. J Neurosurg 114:12881293,2011

\section{Disclosures}

The authors report no conflict of interest concerning the materials or methods used in this study or the findings specified in this paper.

\section{Author Contributions}

Conception and design: Link, Takami. Acquisition of data: Takami. Analysis and interpretation of data: Takami. Drafting the article: Takami, Prummer, Graffeo. Critically revising the article: Link, Takami, Graffeo, Peris-Celda, Giannini, Driscoll. Reviewed submitted version of manuscript: all authors. Approved the final version of the manuscript on behalf of all authors: Link. Statistical analysis: Takami. Administrative/technical/material support: Link, Graffeo, Giannini. Study supervision: Link.

\section{Correspondence}

Michael J. Link: Mayo Clinic, Rochester, MN. link.michael@ mayo.edu. 